Pulsars : Problems \& Progress

ASP Conference Series, Vol. 105,1996

S. Johnston, M. A. Walker and M. Bailes, eds.

\title{
Pulsar radio beams and emission altitudes
}

\author{
Jaroslaw Kijak \\ Max-Planck-Institut für Radioastronomie, Bonn, Germany \\ Astronomy Centre, Pedagogical University, Zielona Góra, Poland \\ Janusz A. Gil \\ Astronomy Centre, Pedagogical University, Zielona Góra, Poland
}

We verify the relationship proposed by Kijak and Gil (1996) for the pulsar radio emission altitudes

$$
r_{K G} \approx 50 \cdot R \nu_{\mathrm{GHz}}^{-0.21} \tau_{6}^{-0.1} P^{0.33},
$$

(see also Eq.3 in Gil \& Krawczyk, 1996), using the pulse-profile Effelsberg raw data at $1.41 \mathrm{GHz}$. We measured profile pulse-widths at the lowest intensity level corresponding to $0.01 \%$ of the maximum intensity (Fig. 1b), using the polarlog-scale technique (Hankins and Fowler, 1986). We calculated opening angles (Fig. 1a) and emission altitudes (Fig. 1c) assuming that: $i$ ) pulsar radiation is narrow-band with radius-to-frequency mapping operating in the emission region, ii) pulsar emission is beamed tangentially to the dipolar magnetic field lines, iii) the extreme profile wings originate near or at the last open field lines.

We argue that the emission altitude depends on : pulsar period $P$, frequency $\nu$, pulsar age $\tau_{6}$ (in units of $10^{6}$ years) approximately as $r \approx 50 \cdot R \nu_{\mathrm{GHz}}^{-1 / 5} \tau_{6}^{-1 / 10} P^{1 / 3}$, or

$$
r \approx(55 \pm 5) \cdot R \nu_{\mathrm{GHz}}^{-0.21 \pm 0.07} \tau_{6}^{-0.1 \pm 0.02}
$$

where $R=10^{6} \mathrm{~cm}$ is the neutron star radius. If the above conclusion is correct then the magnetic field in the emission regions is about $10^{7}-10^{8} \mathrm{G}$, independent of pulsar period $P$ (Fig. 1d). Thus, the emission region is located close to the neutron star in short period pulsars and further away in longer period pulsars. However, the entire pulsar emission region lies within about 100 stellar radii, in consistency with independent estimates (e.g. Cordes 1992).

Acknowledgments. We would like to thank M. Kramer for help during analyzing data and helpful discussion. We also thank $O$. Doroshenko for unpublished data of PSR B1855+09. 

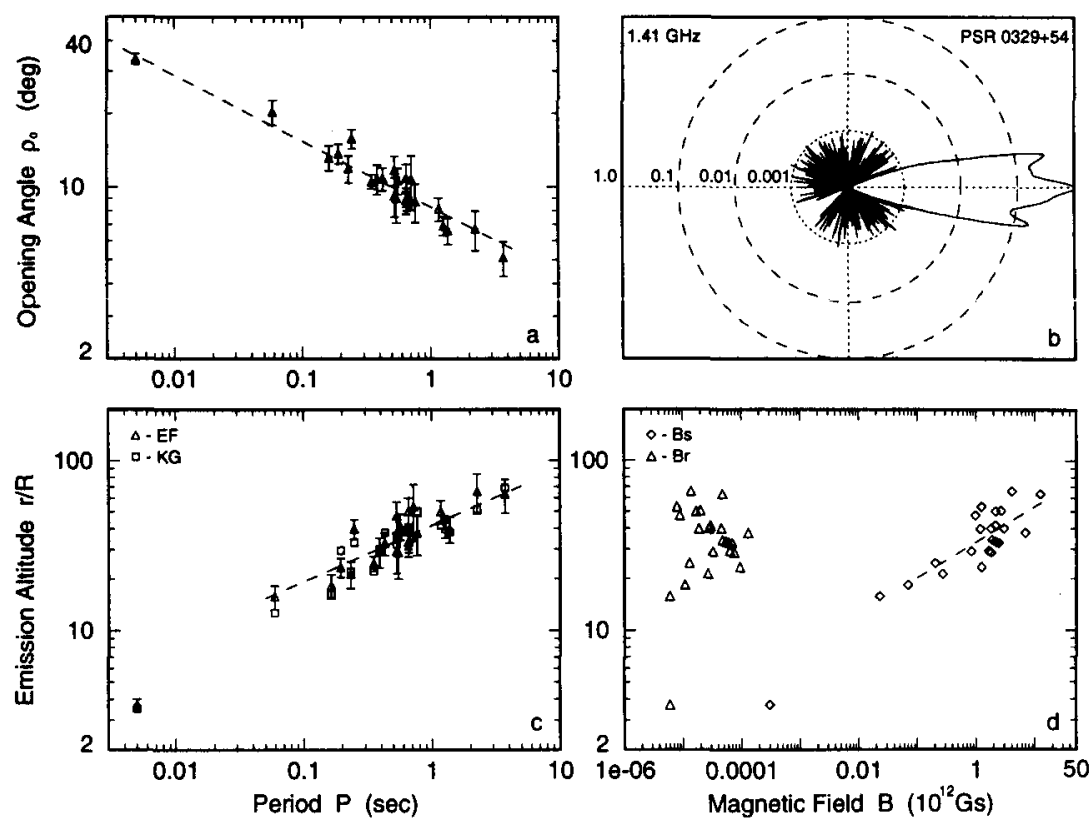

Figure 1. (a) Plot of the opening angle $\rho_{0}$ corresponding to the low intensity profile width $W_{0}(0.01 \%$ of maximum intensity corresponding to the last open field lines) versus pulsar period $P$. The formal fit is $\rho_{0}=(8.3 \pm 0.2) P^{-0.27 \pm 0.03}$. (b) Plot of the profile PSR B0329+54 in the log-scale and the polar co-ordinates. (c) Plot of the emission altitude $r / R$ versus pulsar period $P$ for the Effelsberg data (triangles) and values following from the equation 1 (squares). The formal fit is $r=(42 \pm 2) \cdot R P^{0.33 \pm 0.04}$. (d) Plot of the emission altitude $r / R$ versus the surface magnetic field $B_{s}$ (diamonds). The formal fit is $r=(33 \pm 2) \cdot R B_{s}^{0.21 \pm 0.05}$. A vertical set of triangles represents the magnetic field in the emission region $B_{r}=B_{s}(r / R)^{-3}$. Notice that $B_{r}$ is about $10^{7}-10^{8} \mathrm{G}$ for all pulsars from our sample.

\section{References}

Cordes, J.M. 1992, Proc. IAU Colloquium \# 128, p. 253

Gil, J. \& Krawczyk, A. 1996, MNRAS, in press

Hankins, T.H. \& Fowler, L.A. 1986, ApJ, 304, 256

Kijak, J. \& Gil, J. 1996, in preparation 